\title{
Features of Loss of Stability of the Work of Two-Link Mechanisms That Have an Infinite Number of Degrees of Freedom
}

\author{
Leonid Kondratenko \\ Central Research Institute of Machine Building Technology, Moscow, Russia \\ E-mail: kondrat.leonid@yandex.ru \\ Lubov Mironova \\ Russian University of Transport (MIIT), Moscow, Russia \\ Corresponding author: mironova_lub@mail.ru
}

(Received May 18, 2018; Accepted July 1, 2018)

\begin{abstract}
Mechanisms consisting of two links, a leading (engine) and a slave (executive body), which are connected by long lines of force, are considered. With the use of the new method, general equations are derived in Laplace images describing the oscillations of the velocities of motion and stresses in mechanical and hydraulic systems. Obtained transfer functions. The functional coefficients in these equations take into account the properties of the mechanisms and the distribution of the parameters of the lines of force. As a result of the transition from equations in images to equations in the originals, expressions are obtained that describe in real-time oscillations of the velocities of motion. The criterion for the stability of the work is derived on the basis of Lyapunov's first method. As an example, a volumetric hydraulic drive with long hydraulic lines is considered. With the loss of stability in this mechanism, self-oscillations appeared. Areas of stable and unstable work are defined.
\end{abstract}

Key words- Two-link mechanism, Force line, Distributed parameters, Executive body, System stability, Momentum, Angular momentum, Straight rod, Oscillation frequency, Volumetric hydraulic drive.

\section{Introduction}

Two-link mechanical systems are widely used in industry.

Such systems include:

- mechanical drives (used for mechanical processing of materials during drilling, milling, turning, roller rolling of heat exchange pipes, drilling wells by the rotary method of pipes, etc.);

- hydraulic drives (used in control systems, for drilling wells with the help of hydraulic motors, etc.), as well various devices that in one form or another include two-link mechanisms.

Such mechanisms contain a leading (input) link-source of energy (motor, pump) and a slave (output) link-executive body of the mechanism (cutting part, chisel, etc.) connected by a force line. In addition, in a number of processes (drilling, cylindrical boring, drilling of wells), feedback is not used.

In the case of the application of long force lines, similar systems, following Babakov (1958), can be referred to systems with an infinite number of degrees of freedom. In connection with the fact that in mechanisms with long lines there are significant oscillatory phenomena, it is very important to find a way to calm such oscillations. This largely comes down to the task of ensuring the sustainability of the work. 
International Journal of Mathematical, Engineering and Management Sciences

Vol. 3, No. 4, 315-334, 2018

https://dx.doi.org/10.33889/IJMEMS.2018.3.4-023

The problem of the stability of the motion of mathematics and mechanics has been studied since the 19th century.

To solve such problems using the criteria and theories of Routh (1884), of Hurwitz (1895), of Mihaylov (1939), Chetaev (1950), of Bolotin (1956), of Popov (1973), of Nyquist (1982), of Lyapunov (1992), and etc.

In engineering very rare cases, occur when the calculation of vibrations (fluctuations) of a particular structural element can be performed quite accurately. As a rule, technical calculations are approximate. The main assumptions are made when choosing the calculation scheme of the design. In this case, the insignificant features of the system are ignored and only its main parameters that determine the nature of the phenomenon are singled out.

Systems with distributed masses are in many cases replaced by systems where the masses are concentrated at points. Parts of complex geometric shapes (springs, crankshafts, etc.) are usually reduced to an equivalent straight bar, nonlinear elastic elements are often replaced by equivalent linear elements, and etc.

In a number of cases, such problems are solved by an approximate method with the replacement of the corresponding functional equations by suitable finite-dimensional difference schemes. Examples of such solutions are presented in the works of Godunov and Ryabenkij (1977), Berezansky and Kondratev (1988). As a result, we obtain a problem of optimal control of an approximating system described by equations in finite differences or a system of ordinary differential equations. In this case for a finite-dimensional system, it becomes necessary to consider the maximum principle and to estimate the convergence of approximating controls. However, the questions of the justification for such finite-difference approximation have not yet been investigated sufficiently.

On the stability of systems with distributed parameters not covered by feedback, the authors of the Encyclopedia "Mechanical Engineering" (2000) suggest judging by the dispersion relationsthe intrinsic properties of the physical process. If, however, the differential equations have variable coefficients, then the solution should be sought by a numerical method.

In the works of Siratsetdinov (1987), Shaginyan (2009) the condition of stable operation of the system with distributed parameters is presented. For the general case the condition is formulated as follows:

if in the subspace $W_{[\varphi]}=0$ the process $\varphi \equiv 0$ is stable under integrally small perturbations with respect to the measure $\|\rho\|$, and in the subspace $W_{[\varphi]}<0$ - asymptotically stable under integrally small perturbations with measure $\|\rho\|$, then in a neighborhood $Z_{R}$ for any $\delta\left(\varepsilon, t_{0}\right)>0$ there exists a number such that for $t \geq T$ it is true $\rho[\varphi(\cdot, t)]<2 \delta$, if $\rho\left[\varphi\left(\cdot, t_{0}\right)\right]<\delta$ and. $\rho[h(x)]<\delta$. Here $\varphi$ are the parameters of the process; $h(x)$ is a vector function of admissible solutions.

Publications devoted to the investigation of the stability of open two-link mechanical systems with distributed parameters in the presence of essential nonlinearities, where fairly clear criteria were set forth, could not be found. 
International Journal of Mathematical, Engineering and Management Sciences

Vol. 3, No. 4, 315-334, 2018

https://dx.doi.org/10.33889/IJMEMS.2018.3.4-023

From the above list, it can be seen that two-link mechanisms play a significant role in the development of the economy. Therefore, the identification of possible areas of unsustainable work and the development of methods that ensure stable operation are of considerable interest. In this connection, the proposed work makes an attempt to consider in more detail the consider sustainability issues of these mechanical systems.

\section{Statement of the Problem}

The effectiveness of these mechanisms is determined by the transmitted power, namely the speed of motion of the executive body and the voltages in the force lines.

When these parameters deviate from rational values, it becomes necessary to study the equations

$$
\frac{d \Omega_{j}}{d t}=\sum_{i=1}^{n} \frac{\partial \Omega_{j}}{\partial \xi_{i}} f_{i},
$$

where $\zeta_{i}$ are the coordinates of the system; $f_{i}=d \zeta_{i} / d t ; t$ is the time; $\Omega_{j}$ is the speed of the technological object.

Then the investigation reduces to solving equations

$$
\frac{d \xi_{i}}{d t}=f_{i}\left(t, \xi_{1}, \xi_{2}, \ldots, \xi_{n}\right)
$$

If this seems natural to specialists in control systems, then for the specialists in mechanics this may seem unusual, because they often solve the problem of determining the change in coordinates, the shape of oscillations (the works of Lur'e (1970), Grigolyuk (1973) and Guz' (1985) etc.). Such processes are usually studied by the methods of elasticity theory. For example, Babakov's paper (1958) uses the equation

$v \frac{\partial^{2} u}{\partial t^{2}}-\frac{\partial}{\partial x}\left(E f \frac{\partial u}{\partial x}\right)=Q(x, t)$

whose solution is sought in the form

$$
u(x, t)=\sum_{i=1}^{\infty} H_{i} \theta(x) \sin \left(p_{i} t+\alpha_{i}\right)
$$

where $v, E$ mass and elastic characteristics of the mechanical highway; $f$ cross-sectional area; $Q$ the intensity of external load; $H_{i}, \theta, p_{i}, \alpha_{i}$ constants determined from the initial conditions.

In the case of using the Lagrange equation of the second kind

$$
\frac{d}{d t}\left(\frac{\partial T}{\partial \dot{q}}\right)-\frac{\partial T}{\partial q}=Q ; Q=-\frac{d U}{d q}
$$


International Journal of Mathematical, Engineering and Management Sciences

Vol. 3, No. 4, 315-334, 2018

https://dx.doi.org/10.33889/IJMEMS.2018.3.4-023

We consider the vibrations of the kinetic $(T)$ and potential energy $U$, where $q$ is the generalized coordinate are considered.

The equations are given, which underlie many works on the dynamics of machines, allow us to estimate the change in the displacement of a section of a rod, pipe column, etc. in time and space under given boundary conditions. However, such information, when it is necessary to take into account the interconnection of a large number of factors, is superfluous on the one hand, since it is often enough to know under what conditions the self-oscillations arise of the rotation frequency of the technological object (the executive body) take place, i.e. stability is lost, and under what conditions is not. On the other hand, because of the lack of information on the stresses developed in the dynamic process, it is difficult to assess the likelihood of the destruction of the part or the disturbance of the work process. In the case of using systems with long force lines, there arises the problem of describing the behavior of the mechanism under standard actions, for example, step disturbances, etc.

In the above methods for solving problems, a linear dependence of the voltage on the movement of particles in the material is assumed and a corresponding recalculation is made. However, this is not always justified, since it is known from rheology that the elastic modulus can depend on the oscillation frequency (Reiner 1958, Postnikov 1974). In addition, depending on the stresses, the stem of a drill for deep boring or the pipe string for a well boring can flex and thereby change the features of the formation of force factors on the executive body and the indicators of the work of the mechanism. In this case, a significant influence on the functioning of the system has various non-linear effects, including essential nonlinearity ones.

In this connection, it became necessary to develop a method that explicitly takes into account the vibrations in the velocities of motion and stresses, as well as the influence of various nonlinearities.

\section{Derivation of the Initial Equations}

To solve this problem, when considering longitudinal oscillations in a straight solid rod, in the absence of mass forces, the equation of momentum in differential form is used (Sedov, 1970)

$\rho \frac{\partial v}{\partial t}=\frac{\partial \sigma}{\partial x}$

and the equation of longitudinal oscillations

$\frac{\partial^{2} u}{\partial t^{2}}=\frac{E}{\rho} \frac{\partial^{2} u}{\partial x^{2}}$.

Here $v$ is the velocity of the longitudinal displacement of the point in the considered crosssection; $u$ is the displacement along the $x$ axis; $\sigma$ is the longitudinal (normal) stress; $\rho$ is the density of the material; $E$ is the elastic modulus (Young).

The quantity $v$ is defined as the rate of change of the function $u$

$v=\frac{\partial u}{\partial t}$. 
International Journal of Mathematical, Engineering and Management Sciences

Vol. 3, No. 4, 315-334, 2018

https://dx.doi.org/10.33889/IJMEMS.2018.3.4-023

From the equation (1) we define the value of $\partial v / \partial t$ and substitute it into equation (2). We obtain

$$
\begin{aligned}
& \frac{1}{\rho} \frac{\partial \sigma}{\partial x}=\frac{E}{\rho} \frac{\partial^{2} u}{\partial x^{2}} \\
& \text { or } \\
& \frac{1}{E} \frac{\partial \sigma}{\partial x}=\frac{\partial^{2} u}{\partial x^{2}} .
\end{aligned}
$$

We integrate the result obtained (4) with respect to $x$, assuming that for $x=0, \sigma=$ const

$$
\frac{1}{E} \int_{0}^{x} \frac{\partial \sigma}{\partial x} d x=\int_{0}^{x} \frac{\partial^{2} u}{\partial x^{2}} d x
$$

The solution of relation (5) will be

$$
\frac{1}{E}\left(\sigma_{x}-\sigma_{0}\right)=\left(\frac{\partial u}{\partial x}\right)_{x}-\left(\frac{\partial u}{\partial x}\right)_{x=0}
$$

We differentiate relation (6) with respect to time $t$, taking into account expression (3), we obtain $\frac{1}{E} \frac{\partial \sigma_{x}}{\partial t}=\frac{\partial v}{\partial x}$

Let's denote the current value of the stress $\sigma_{x}$ by the letter " $\sigma$ ". Taking into account that the surface forces acting on each point of the section of the elementary volume are directed in the opposite direction from the direction of the velocity of motion, the equation obtained (7) and equation (1) can be rewritten in the form

$\rho \frac{\partial v}{\partial t}=-\frac{\partial \sigma}{\partial x}$
$\frac{1}{E} \frac{\partial \sigma}{\partial t}=-\frac{\partial v}{\partial x}$

When considering torsion oscillations, we assume that there is no deplanation of the cross sections. Then the elastic vibrations are described by the equation

$$
\frac{\partial^{2} \varphi}{\partial t^{2}}=\frac{G}{\rho} \frac{\partial^{2} \varphi}{\partial x^{2}}
$$

where $\varphi, x$ respectively, are the angle of rotation of the section of the force line and the coordinate with zero in the center of the leading end of the drive; $G$ is the shear modulus of the material; $\rho$ is the density of the material; $t$ is the time.

Let us compose the equation of angular momentum for a finite volume of a continuous medium in the absence of viscous friction in the material (Sedov, 1970) 
International Journal of Mathematical, Engineering and Management Sciences

Vol. 3, No. 4, 315-334, 2018

https://dx.doi.org/10.33889/IJMEMS.2018.3.4-023

$\frac{d \int_{V} \vec{K} \rho d \delta}{d t}=\int_{S} \vec{\theta}_{s} d f$.

Here $\vec{K}$ vector, the modulus of the vector $|\vec{K}|$ is the density of the intrinsic angular momentum; $\vec{\theta}_{s}$ vector, moment of distribution of surface pairs (here only pairs in cross-section); $d \delta$ an infinitesimal volume; $S$ area of the bounding surface of a body of volume $V$; $d f$ infinitesimal surface area of $S$.

We write down the formula for the modulus of the vector $|\vec{K}|$

$|\vec{K}|=r^{2} \Omega$.

Here, $r$ is the radial coordinate; $\Omega$ angular velocity.

When rotating around its own axis, the rod transmits the torque

$M=\int_{S} \vec{\theta}_{s} d f=\int_{S} r \tau d f$,

where $\tau$-tangential stresses when torsion.

Since $\vec{K}$ and $\vec{\theta}_{s}$ are vectors, so applying the Gaus-Ostrogradsky theorem, we obtain $\int_{S} \vec{K} d f=\int_{V} \operatorname{div} \vec{K} d \delta ; \quad \int_{S} \vec{\theta}_{s} d f=\int_{V} \operatorname{div} \vec{\theta}_{s} d \delta$

We express the vector $\vec{\theta}_{s}$ in terms of the direction cosines, we obtain $\vec{\theta}_{s}=B \cos (\vec{n}, x)+Q \cos (\vec{n}, y)+R(\vec{n}, z)$,

where $\vec{n}$ is normal to the surface $S ; x, y, z$ are the coordinate axes.

Then in our case

$\int_{V} \operatorname{div} \vec{\theta}_{s} d \delta=\int_{V}\left(\frac{\partial B}{\partial x}+\frac{\partial Q}{\partial y}+\frac{\partial R}{\partial z}\right) d \delta=\int_{V}\left(\frac{\partial(r \tau)}{\partial x}\right) d \delta$.

We substitute (13) into (11), taking into account (12) we obtain $\frac{d \int_{V} r^{2} \Omega \rho d \delta}{d t}=\int_{V} \frac{\partial(r \tau)}{\partial x} d \delta ; \quad \Omega=\frac{\partial \varphi}{\partial t}$.

Whence the equation of the moments of momentum in the differential form, when the positive direction of rotation of the shaft is taken against the clockwise movement, we write in the form 
International Journal of Mathematical, Engineering and Management Sciences

Vol. 3, No. 4, 315-334, 2018

https://dx.doi.org/10.33889/IJMEMS.2018.3.4-023

$\frac{\partial\left(r^{2} \Omega \rho\right)}{\partial t}=-\frac{\partial(r \tau)}{\partial x}$

or for an elementary part of the rod with an outer radius $r$ at $\rho=$ constant

$\rho r \frac{\partial \Omega}{\partial t}=-\frac{\partial \tau}{\partial x}$

where $\tau$ maximum tangential stress.

From a comparison of (10) and (15) we arrive at the equation

$G \frac{\partial^{2} \varphi}{\partial x^{2}}=-\frac{1}{r} \frac{\partial \tau}{\partial x}$

We integrate equation (16) in $x$. We obtain

$r G \frac{\partial \varphi}{\partial x}=\left.r G \frac{\partial \varphi}{\partial x}\right|_{0}-\left(\tau_{x}-\tau_{0}\right)=-\tau+B_{\tau}$

Here $B_{\tau}-$ is a constant characterizing the stresses at $x=x_{0}, t=t_{0}$.

We differentiate equation (17) in $t$. We obtain

$r G \frac{\partial \Omega}{\partial x}=-\frac{\partial \tau}{\partial t}$.

The system of equations (15), (18) makes it possible to describe changes in tangential stresses on the outer cylindrical surface in an elementary volume and of the speed of movement of elementary sections of the line.

Let us turn now to longitudinal oscillations. Assuming $E, \rho$ to be constant and conducting a onedimensional Laplace transform of these equations under zero initial conditions (Ivanov et al., 1971), we obtain

$\rho s u(s)=-\frac{d \sigma(s)}{d x}$

$E \frac{d u(s)}{d x}=-s \sigma(s)$.

Here $s$ is a complex variable: $s=j \omega$, where $j$ is the imaginary unit; $\omega$ is the circular oscillation frequency.

The solution of the system of equations (19), (20) allows us to find for the selected section the instantaneous deviations from the steady-state values of the voltage and the velocity of the rod segments. Each of these quantities will be the sum of the quantities of the same name with it, determined in the front of the perturbation, propagating in the forward and backward directions.

The instantaneous deviations of the marked variables, as well as the speed of propagation of the disturbance along the force line, depending on the physical and geometric properties of the line. This is reflected in the operator-coefficient of wave propagation 
International Journal of Mathematical, Engineering and Management Sciences

Vol. 3, No. 4, 315-334, 2018

https://dx.doi.org/10.33889/IJMEMS.2018.3.4-023

$\theta(s)= \pm s \sqrt{\frac{\rho}{E}}$

which characterizes the dynamic features of the lines with the parameters distributed along the length.

Differentiating equation (19) with respect to $x$, eliminating the derivative $d v(s) / d x$ with the aid of (20) and applying (21), we obtain

$\frac{\partial^{2} \sigma(s)}{\partial x^{2}}-\theta^{2}(s) \sigma(s)=0$.

This equation is a second-order differential equation with constant coefficients. The solution has the form

$\sigma(s, x)=C_{1} \exp [\theta(s) x]+C_{2} \exp [-\theta(s) x]$.

The constants of the integrals $C_{1}, C_{2}$ are determined by the boundary conditions (Kondratenko, 2005):

$x=0, \sigma(s, x)=\sigma_{1}(s, 0)$,

$\frac{\partial \sigma(s, x)}{\partial x}=-\frac{1}{s} E v_{1}(s, 0) \theta^{2}(s)$.

The last condition in (24) was obtained from (19) by replacing $\rho s=\frac{1}{s} E \theta^{2}(s)$.

Then, taking (24) into account, we obtain

$$
\begin{aligned}
& C_{1}=\frac{1}{2}\left[\sigma_{1}(s, 0)-\frac{1}{s} E \mathrm{v}_{1}(s, 0) \theta(s)\right] \\
& C_{2}=\frac{1}{2}\left[\sigma_{1}(s, 0)+\frac{1}{s} E \mathrm{v}_{1}(s, 0) \theta(s)\right] .
\end{aligned}
$$

Taking (23) and (25) into account, we obtain the solution

$\sigma(s, x)=\frac{1}{2}\left\{\sigma_{1}(s, 0)[\exp (\theta(s) x)+\exp (-\theta(s) x)]-\frac{1}{s} E \mathrm{v}_{1}(s, 0) \theta(s)[\exp (\theta(s) x)-\exp (-\theta(s) x)]\right\}$

or after the introduction of hyperbolic functions

$\sigma(s, x)=\sigma_{1}(s, 0) \operatorname{ch}[\theta(s) x]-\frac{1}{s} E \mathrm{v}_{1}(s, 0) \theta(s) \operatorname{sh}[\theta(s) x]$.

Solving the system of equations (19), (20) with respect to $v(s, x)$ by the above method, we obtain $\frac{\partial^{2} v(s)}{\partial x^{2}}-\theta^{2}(s) v(s)=0$.

For the boundary conditions $x=0$, 
International Journal of Mathematical, Engineering and Management Sciences

Vol. 3, No. 4, 315-334, 2018

https://dx.doi.org/10.33889/IJMEMS.2018.3.4-023

$\mathrm{v}(s, x)=\mathrm{v}_{1}(s, 0)$,

$\frac{\partial v(s, x)}{\partial x}=-\frac{1}{E} s \sigma_{1}(s, 0)$.

We finally obtain

$v(s, x)=v_{1}(s, 0) \operatorname{ch}[\theta(s) x]-\frac{1}{E} \frac{s \sigma_{1}(s, 0)}{\theta(s)} \operatorname{sh}[\theta(s) x]$.

If the supplied moment of momentum does not pass through the output link, to waves of disturbance are reflected from the end of the lines. We will consider the case of an agreed load, when there are no reflected waves in the system. In this case, the boundary conditions are the following relations:

$\mathrm{v}(s, l)=\mathrm{v}_{2}(s), \mathrm{v}(s, 0)=\mathrm{v}_{1}(s), \sigma(s, l)=\sigma_{2}(s), \sigma(s, 0)=\sigma_{1}(s)$.

$(1-c) \sigma_{2}(s)=\frac{1}{f_{2}}\left[F(s)+h \mathrm{v}_{2}(s)+m s \mathrm{v}_{2}(s)\right]$.

Here $f_{2}$ is the cross-sectional area of the force line in front of the executive body of mass $m ; c, h$ are friction loss coefficients proportional to the stress and speed of movement, $F$ is the resistance force acting on the executive body; $l$ is the length of the force line. The coefficient $h$ takes into account only frictional losses proportional to the speed of the load.

We jointly solve (26) - (30), performing the following transformations

$$
\begin{aligned}
& \sigma_{1}(s)=\frac{1}{c h A}\left[\sigma_{2}(s)+\frac{1}{s} E \mathrm{v}_{1}(s) \theta(s) s h A\right] \\
& \mathrm{v}_{2}(s)=\mathrm{v}_{1}(s) \operatorname{ch} A-\frac{s}{E \theta(s)} \operatorname{sh}\left\{\frac{1}{\operatorname{ch} A}\left[\sigma_{2}(s)+\frac{1}{s} E \mathrm{v}_{1}(s) \theta(s) s h A\right]\right\} \\
& \mathrm{v}_{2}(s)=\frac{\mathrm{v}_{1}(s)}{c h A}-\frac{s}{E \theta(s)} \sigma_{2}(s) \operatorname{th} A \\
& (1-c)\left[\frac{\mathrm{v}_{1}(s)}{\operatorname{ch} A}-\mathrm{v}_{2}(s)\right] \frac{E \theta(s)}{s t h A}=\frac{1}{f_{2}}\left[F(s)+h \mathrm{v}_{2}(s)+m s \mathrm{v}_{2}(s)\right] \\
& \mathrm{v}_{2}(s)\left[1-c+h \vartheta(s) s+m \vartheta(s) s^{2}\right]=\frac{(1-c) \mathrm{v}_{1}(s)}{c h A}-F(s) \vartheta(s) s
\end{aligned}
$$

Here

$$
\vartheta(s)=\vartheta_{0} Z(s) ; \vartheta_{0}=\frac{l}{E f_{2}} ; Z(s)=\frac{t h A}{A} ; A=l \theta(s) .
$$

Having performed similar transformations with respect to torsion oscillations, we obtain

$$
\Omega_{2}(s)\left[1+h_{k} \vartheta_{k}(s) s+J \vartheta_{k}(s) s^{2}\right]=\frac{\Omega_{1}(s)}{c h A_{k}}-M_{r}(s) \vartheta_{k}(s) s .
$$


International Journal of Mathematical, Engineering and Management Sciences

Vol. 3, No. 4, 315-334, 2018

https://dx.doi.org/10.33889/IJMEMS.2018.3.4-023

Here $\Omega_{1}, \Omega_{2}$ the angular speeds of rotation of the input and output shafts the speed of the output shaft; $M_{r}$ moment of resistance. The subscript $k$ refers to torsion oscillations

For the tube of formula (32), we take in the following form

$$
\begin{aligned}
& \vartheta_{k}(s)=\vartheta_{k 0} Z_{k}(s) ; \quad \vartheta_{k 0}=\frac{l}{r G W} ; \quad W=\frac{\pi\left(r^{4}-r_{0}^{4}\right)}{2 r} \quad Z_{k}(s)=\frac{t h A_{k}}{A_{k}} ; \quad A_{k}=l \theta(s) ; \\
& \theta(s)= \pm s \sqrt{\frac{\rho}{G}} .
\end{aligned}
$$

Here $G$ - is the shear modulus; $W$ is the polar moment of inertia; $r_{0}, r$ inner and outer radius of the pipe.

For a volume hydraulic drive, after similar transformations, was received (Adamenko and Kondratenko, 2001; Kondratenko, 2008)

$$
\begin{aligned}
& \Omega(s)\left\{J \vartheta(s) s^{2}+\left[J \tau+h_{h} \vartheta_{h}(s)\right] s+(1-c)+h_{h} \tau\right\}=(1-c) Q(s) \psi(s)-M_{r}(s)\left[\vartheta_{h}(s) s+\tau\right] \\
& \vartheta_{h}(s)=\frac{\vartheta_{h 0}(s)}{E_{r}(s)} Z_{h}(s) .
\end{aligned}
$$

Here $\Omega$ is the speed of the output shaft; $J$ is the moment of inertia of the rotating parts; $\tau$ is criterion of tightness; $h_{h}$ is coefficient of friction loss proportional to the speed of motion; $\vartheta_{h 0}$ is elasticity coefficient; $c$ is coefficient of friction loss proportional to the pressure drop; $M_{r}$ is the moment of resistance; $E_{r}(s), \psi(s)$ are polynomials obtained under transformations; $w$ volumetric constant of the hydraulic motor; $Q$ is pump flow rate.

We use the following formulas

$$
\tau=\frac{K}{w^{2}} ; \vartheta_{h 0}=\frac{l f_{1}}{k w^{2}} ; \theta_{01}(s)=s \sqrt{\frac{\rho_{0}}{\kappa}}, Z_{h}(s)=\frac{t h A_{h}}{A_{h}} ; A_{h}=l \theta_{01}(s) \text {. }
$$

Here $l$ is the length of the force line; $f_{1}$ is the cross-sectional pressure line; $\theta_{01}(s)$ is operatorcoefficient wave propagation in the pressure line; $\rho_{0}, \kappa$ respectively, the density and modulus of elasticity of the liquid. The subscript $h$ refers to the hydraulic motor.

\section{The Solution of the Problem}

Lyapunov (1935) considered two methods of studying stability.

The first method. The methods that lead to the determination of the general and particular solution of differential equations are investigated.

The second method. Without determining the solutions of differential equations, it is required to find a function of generalized coordinates and time, the full derivative of which, by virtue of the equations of motion under consideration, possesses certain properties (the Lyapunov function). 
The wider application of the second method, as of the most convenient method, led to the use of linearized differential equations with constant coefficients and of special criteria of stability proposed by Routh, Hurwitz, Mikhailov, Nyquis, and others. Turning to equations (31) - (36), we see that the coefficients of the characteristic equations, which are multiplied by the variable s, depend on this variable. Since the use of these criteria proved to a difficulty, a new technique for assessing stability was developed using Lyapunov's first method.

Analyzing equations (31) - (36), we see that they are similar. In this connection, we will carry out the following reasoning with the equation for the volume hydraulic drive (34), with which it was easier to perform an oscilloscope recording of the oscillatory process in full-scale experiments.

We decompose the equation (34) algebraically into two equations:

$Q(s) \psi(s)=w \Omega(s)+\tau w^{2} p(s)+w^{2} \vartheta_{h}(s) p(s) s ;$

$(1-c) w p(s)=M_{r}(s)+h_{h} \Omega(s)+J \Omega(s) s$,

where $p-$ is the pressure drop across the hydraulic motor.

The correctness of the decomposition is easily verified by the inverse solution.

As follows from equation (34), the coefficients that are multiplied by the variables s depend on the function (35).

We substitute in $Z_{h}(s)$ the variable

$\theta_{01}(s)=s \sqrt{\frac{\rho_{0}}{\kappa}}=j \omega \sqrt{\frac{\rho_{0}}{\kappa}}$.

We obtain

$Z_{h}(j \omega)=\frac{\operatorname{th}(\operatorname{lj} \omega) \sqrt{\rho_{0} / \kappa}}{l j \omega \sqrt{\rho_{0} / \kappa}}=\frac{j \operatorname{tg} \alpha}{j \alpha}=\frac{\operatorname{tg} \alpha}{\alpha} ; \alpha=l \omega \sqrt{\frac{\rho_{0}}{\kappa}}$.

Here $\omega$ is the circular oscillation frequency.

The function of the complex variable of formula (36) becomes real. Taking (38) into account, we have

$Z_{h}(\alpha)=\frac{\operatorname{tg} \alpha}{\alpha} ; \vartheta_{h}(\alpha)=\frac{\vartheta_{h 0}}{E_{r}(\omega)} Z_{h}(\alpha)$.

The graph of the function $Z_{h}(\alpha)$ is shown in Fig. 1. 
International Journal of Mathematical, Engineering and Management Sciences

Vol. 3, No. 4, 315-334, 2018

https://dx.doi.org/10.33889/IJMEMS.2018.3.4-023

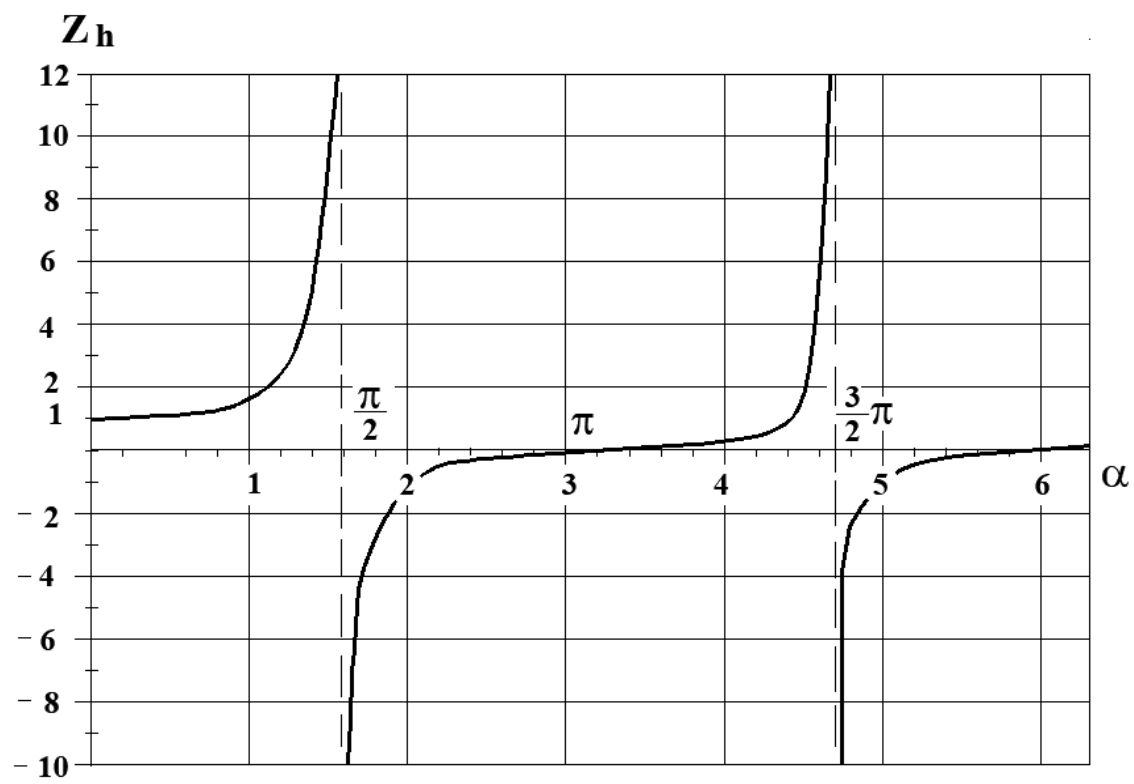

Fig. 1. Change of the function $Z_{h}$ depending on the dimensionless parameter $\alpha$

The complex functions $\psi(s)$ and $E_{r}(s)$ that belong to (37) are replaced by the real parts $\psi(\omega)$ and $\vartheta(\alpha)$, which essentially exceed on modulus practically over the entire frequency range the value of the imaginary parts of these functions.

Passing to the originals of equations (37), we perform the inverse Laplace transform of the functions

$$
F_{1}(s)=w^{2} \vartheta_{h}(\alpha) p(s) s, F_{2}(s)=\psi(\omega) Q(s) .
$$

Then

$$
\begin{aligned}
& L^{-1}\left[F_{1}(s)\right]=\frac{1}{2 \pi j} \int_{c-j \omega}^{c+j \omega} w^{2} \vartheta_{h}(\alpha) p(s) s e^{s t} d s=w^{2} \vartheta_{h}(\alpha) \frac{d p}{d t} ; \\
& L^{-1}\left[F_{2}(s)\right]=\frac{1}{2 \pi j} \int_{c-j \omega}^{c+j \omega} \psi(\omega) Q(s) e^{s t} d s=\psi(\omega) Q(t) .
\end{aligned}
$$

Then the originals of equations (36) can be written in the form

$$
Q(t) \psi(\omega)=w \Omega(t)+\tau w^{2} p(t)+w^{2} \vartheta_{h}(\alpha) \frac{d p}{d t}
$$

$(1-c) w p(t)=M_{r}(t)+h_{h} \Omega(t)+J \frac{d \Omega}{d t}$. 
International Journal of Mathematical, Engineering and Management Sciences

Vol. 3, No. 4, 315-334, 2018

https://dx.doi.org/10.33889/IJMEMS.2018.3.4-023

The permissibility of using equations (38) for studying the dynamic properties of the systems under consideration was verified by constructing the frequency characteristics of a volumetric hydraulic drive in various ways (Adamenko and Kondratenko, 2001; Kondratenko, 2008): calculation of frequency characteristics by equations (34), (36); calculation of frequency characteristics using the fourth-order Runge-Kutta method and from the full-scale experiment.

We now introduce the operator $D \equiv d / d t$ and transform the system (39)

$\frac{(1-c)}{\left(1-c+\tau h_{h}\right) w} Q(t) \psi(\omega)-\frac{\tau+\vartheta_{h}(\alpha) D}{1-c+\tau h_{h}} M_{r}(t)=\Omega(t)\left(1+2 \zeta T D+T^{2} D^{2}\right)$,

where

$2 \zeta T=\frac{J \tau+h \vartheta_{h}(\alpha)}{1-c+\tau h_{h}} ; T=\sqrt{\frac{J \vartheta_{h}(\alpha)}{1-c+\tau h_{h}}} ; \zeta=0,5\left(\frac{\tau}{\vartheta_{h}(\alpha)}+\frac{h_{h}}{J}\right)$.

In formulas (40) $T$ is the time constant; $\zeta$ is the damping factor.

If $M_{r}=$ const, then equation (40), (41) can be rewritten

$k Q(t)=\frac{\tau}{1-c+\tau h_{h}} M_{r}+\Omega(t)\left(1+2 \zeta T D+T^{2} D^{2}\right)$,

where $k=\frac{1-c}{\left(1-c+\tau h_{h}\right) w} \psi(\omega)$.

We introduce the concepts static error $\frac{\tau M_{r}}{1-c+\tau h_{h}}$, and reduced velocity $\Omega_{S}=\frac{\Omega+\tau M_{r}}{1-c+\tau h_{h}}$.

Then (42) can be rewritten in the form

$k Q(t)=\Omega_{S}(t)\left(1+2 \zeta T D+T^{2} D^{2}\right)$.

Equation (43) describes the dynamics of the system in deviations from a certain static regime at $Q$ $(t)=Q_{0}$ and a moment of resistance $M_{r}(t)=M_{r 0}$ with a steady-state rotation frequency $\Omega=\Omega_{0}$.

Most mechanical systems operate under conditions where at least on one of the inputs (outputs) a periodic signal acts. Quite often, such a signal can be represented in the form of a harmonic function. Thus, for example, when the shaft of an axial-piston hydraulic motor is rotated then due to the uneven friction in one of the pistons, there may be a somewhat increased frictional force. Then for one revolution there will be one surge of resistance to rotation, i.e. in the considered steady-state regime, the moment oscillates (vibrates) with a small amplitude according to the law

$M_{r}(t)=M_{r 0}\left[1+b \sin \left(a \Omega_{0} t\right)\right]$.

In expression (44), the introduced factor " $a$ " takes into account the possibility of generating oscillations from other pistons of the hydraulic motor. 
International Journal of Mathematical, Engineering and Management Sciences

Vol. 3, No. 4, 315-334, 2018

https://dx.doi.org/10.33889/IJMEMS.2018.3.4-023

Consequently, for a known velocity in a linear system there will be stable small oscillations with a frequency

$\omega_{0}=a \Omega_{0}$.

At such a frequency, the coefficients $\psi(\omega), \vartheta_{h}(\alpha)$ will have absolutely definite values $\psi\left(\omega_{0}\right)$, $\vartheta_{h}\left(\alpha_{0}\right)$.

We rewrite equation (43) in Laplace images for the case when there are no deviations from the steady-state operating mode in the system, and we determine the transfer function

$W=\frac{\Omega_{S}(s)}{Q(s)}=\frac{k}{1+2 \zeta T s+T^{2} s^{2}}$.

For a small step-like action $\Delta Q(t)=q_{0} \cdot 1(t)$, when the changes of coefficients can be neglected, the equation of motion in the images will have the form

$\Omega_{S}(s)\left(1+2 \zeta T s+T^{2} s^{2}\right)=\frac{k q_{0}}{s}$.

Using the transition tables (Besekerskij, 1972) from images to originals, the oscillation of the speed of motion can be described by the equation

$\Omega_{s}(t)=k\left\{1-\exp (-\alpha t)\left[\cos (\omega t)+\frac{\alpha \sin (\omega t)}{\omega}\right]\right\}$,

where

$\alpha=\frac{\zeta}{T}=0,5\left[\frac{\tau}{\vartheta_{h}(\alpha)}+\frac{h_{h}}{J}\right] ; \omega=\sqrt{\frac{1-\zeta^{2}}{T^{2}}} ; T=\sqrt{\frac{J \vartheta_{h}(\alpha)}{1-c+\tau h_{h}}}$.

It is seen from (47), (48) that for $\alpha>0$, and therefore $\vartheta_{h}(\alpha)>0$, the quantity $\Omega_{S}$ tends to some steady-state value, that is, the process is stable.

If $\vartheta_{h}(\alpha)<0$ and $\alpha<0$, then the quantity $\Omega_{S}$ tends to infinity, i.e. the process will be unstable.

Let's assume that the hydraulic drive has switched to another stationary mode of operation with the fluid flow rate

$Q(t)=Q_{01}$, at which the steady-state speed $\Omega=\Omega_{01}$. In this case, the coefficients of equation (42) will have other values and the response to a small change in the signal will be other.

With a constant flow rate of the pump under the action of a new value of the resistance moment due to leaks, the output shaft of the hydraulic motor will rotate with another steady-state frequency $\Omega_{01}$. In this case, the coefficients of the characteristic equation (40), (41) and equation (45) will also be different. The average values of these coefficients will be determined by the steady-state frequency $\Omega_{01}$.

It can be seen from the graph in Fig. 1 that periodically $Z$ becomes negative. Since, depending on the pump or static load, the stationary speed of the hydraulic motor will be different, then it is 
International Journal of Mathematical, Engineering and Management Sciences

Vol. 3, No. 4, 315-334, 2018

https://dx.doi.org/10.33889/IJMEMS.2018.3.4-023

possible that in some operating modes the coefficients of the characteristic equation (43) will become be negative. In this case, for a small jump-like change in the perturbing effect, the rotation frequency will either increase infinitely or decrease to zero. Consequently, the system will become be unstable.

If $Z$ tends to infinity, and hence $\vartheta_{h}(\alpha) \rightarrow \infty$, then the damping coefficient $\zeta$ will be determined by the ratio $h / J$, and the time constant $T$ become will tend to infinity.

As an example, consider the frequency response of a volumetric hydraulic drive with long lines, characterizing the effect of oscillations of the moment of resistance on the frequency of rotation of the output shaft, see (34), (35).

$W_{\Omega}(s)=\frac{\Omega(s)}{M(s)}=-\frac{\tau+\vartheta_{h}(s) s}{1-c+\tau h_{h}+\left[J \tau+h_{h} \vartheta_{h}(s)\right] s+J \tau s^{2}}$

In this case, the calculations will be carried out using complex variables according to equation (49).

Hydraulic drive parameters: $w=1,9 * 10^{-3} \mathrm{M}^{3} / \mathrm{rad} ; Q_{0}=0.025 \mathrm{~m}^{3} / \mathrm{s} ; \tau=0.00051 / \mathrm{Nms} ; l=2000$ $\mathrm{m}, \kappa=2000 \mathrm{MPa} ; \rho=10^{3} \mathrm{~kg} / \mathrm{m}^{3}$.

Fig. 2 shows the calculated frequency characteristics of such a system, illustrating the effect of oscillations of the moment of resistance on the oscillations of the speed of the output shaft.

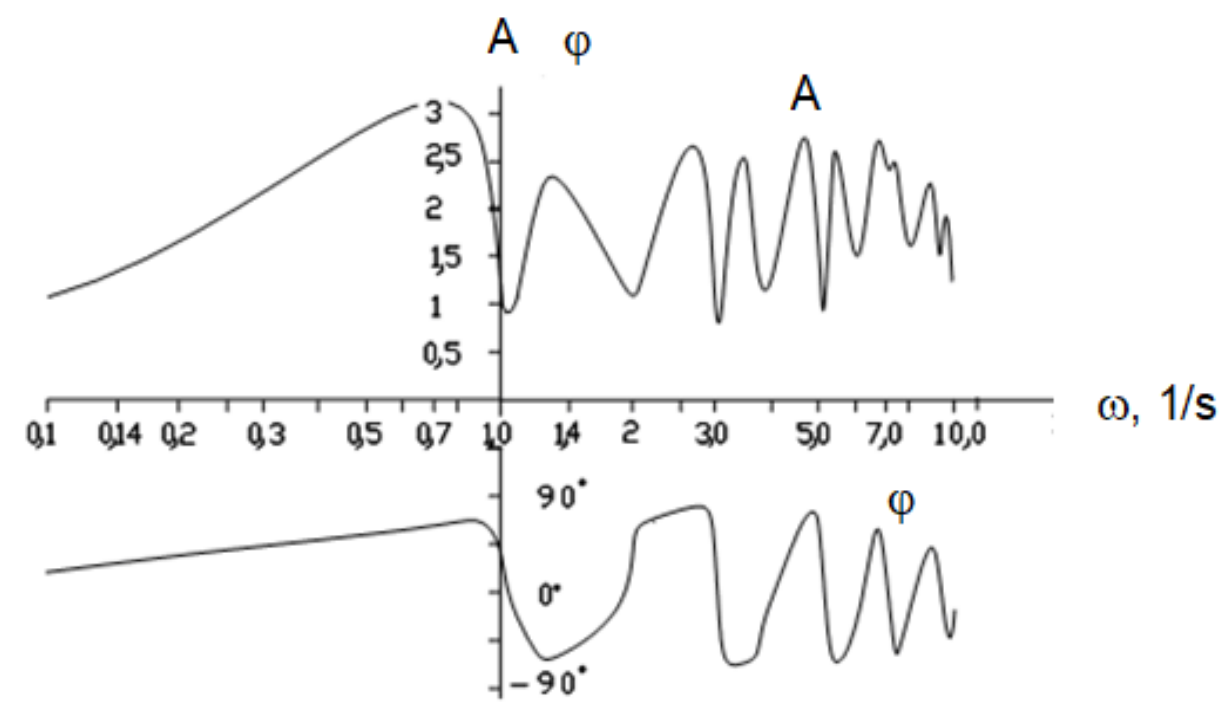

Fig. 2. Amplitude (A) and phase $(\varphi)$ frequency characteristics of a volumetric hydraulic drive 
International Journal of Mathematical, Engineering and Management Sciences

Vol. 3, No. 4, 315-334, 2018

https://dx.doi.org/10.33889/IJMEMS.2018.3.4-023

A dimensionless parameter characterizing the degree of distribution of the system parameters from the indicated data is determined by the expression

$\alpha=l \omega \sqrt{\frac{\rho}{\kappa}}=1,414 \omega$.

From the graph in Fig. 1 it follows that the critical values are $\alpha=1.57 ; 4.71 ; 7.85 \ldots$ These values correspond to frequencies $\omega=1.11 ; 3.34 ; 5.57 \ldots$, for which the function $Z_{h}$ becomes negative.

The frequency characteristics indicate that in the frequency range 1.11-2.22; 3.34-4.41; 5.57-6.62 $\ldots s^{-1}$; the phase of oscillations changes rapidly by $180^{\circ}$.

Similar phenomena can be traced and in mechanical systems.

However, the frequency characteristics do not allow us to reveal the features of the process of loss of stability of the system under consideration.

To solve this problem, we apply the fourth-order Runge-Kutta method (Kamke, 1959).

For this purpose, on the basis of the system (24), we shall construct a system of equations for modeling

$$
\begin{aligned}
& \frac{d \Omega}{d t}=\frac{1}{J}\left[(1-c) w \Delta p(t)-M_{r}(t)-M_{n}(t)\right] ; \\
& \frac{d p_{1}}{d t}=\frac{\kappa_{1}\left[Q(t)-w \Omega(t)-Q_{r}(t)\right]}{V_{1} Z_{1}(\omega)} \\
& \frac{d p_{2}}{d t}=\frac{\kappa_{2}\left[w \Omega(t)+Q_{r}(t)+Q_{r p}(t)\right]}{V_{2} Z_{2}(\omega)}
\end{aligned}
$$

where $p_{1}, p_{2}$ are the pressures in the pressure main and the return line; $Q_{r}, Q_{r p}$ are the leakage of working fluid in the pressure line, through the jet nozzles and through the outlet resistance; $\kappa_{1}, \kappa_{2}$ are the elastic modules of the working (rinsing) liquid in the pressure main and of the return line; $V_{1}, V_{2}$ are the volume of the pressure main and of return line; $M_{n}$ is nonlinear friction.

Due to the fact that the function element (bore bit) has three roller cones, it was assumed that the moment of resistance varies according to the law

$$
M_{r}=M_{r 0}[1+0,005 \sin (3 \Omega t)] \text {. }
$$

At the same time, on the basis of bench experimental data (Kondratenko, 2005), nonlinear friction was calculated from equation

$$
M_{n}=7,6\left[\frac{\Omega}{2 \pi}-0,8\right]^{2}+0,9 \Omega+0,8(\mathrm{Nm}) \text {. }
$$


International Journal of Mathematical, Engineering and Management Sciences

Vol. 3, No. 4, 315-334, 2018

https://dx.doi.org/10.33889/IJMEMS.2018.3.4-023

The calculation was carried out with the help of a PC. The simulation was carried out at a liquid flow rate of the pump $Q=26 \mathrm{l} / \mathrm{sec}$. The solution of the system of equations (50) was carried out using the fourth-order Runge-Kutta method with a constant step of computations. At the same time, non-linear factors (friction, unidirectional rotation) were taken into account in each of the 4 stages of the calculation cycle. And for each introduced frequency of forced oscillations $\omega$, the value of $\alpha$ and the functions $\psi(\omega), \vartheta_{s}(\alpha)$ were recalculated.

The simulation was carried out when is step of computations $\mathrm{H}=10^{-4} ; 2 * 10^{-4} \mathrm{sec}$. The error did not exceed $10 \%$. The moment of resistance changed each time in a stepwise manner by an amount of $\Delta M_{r}=1 \mathrm{kNm}$.

Some simulation results are shown in Fig. 3.

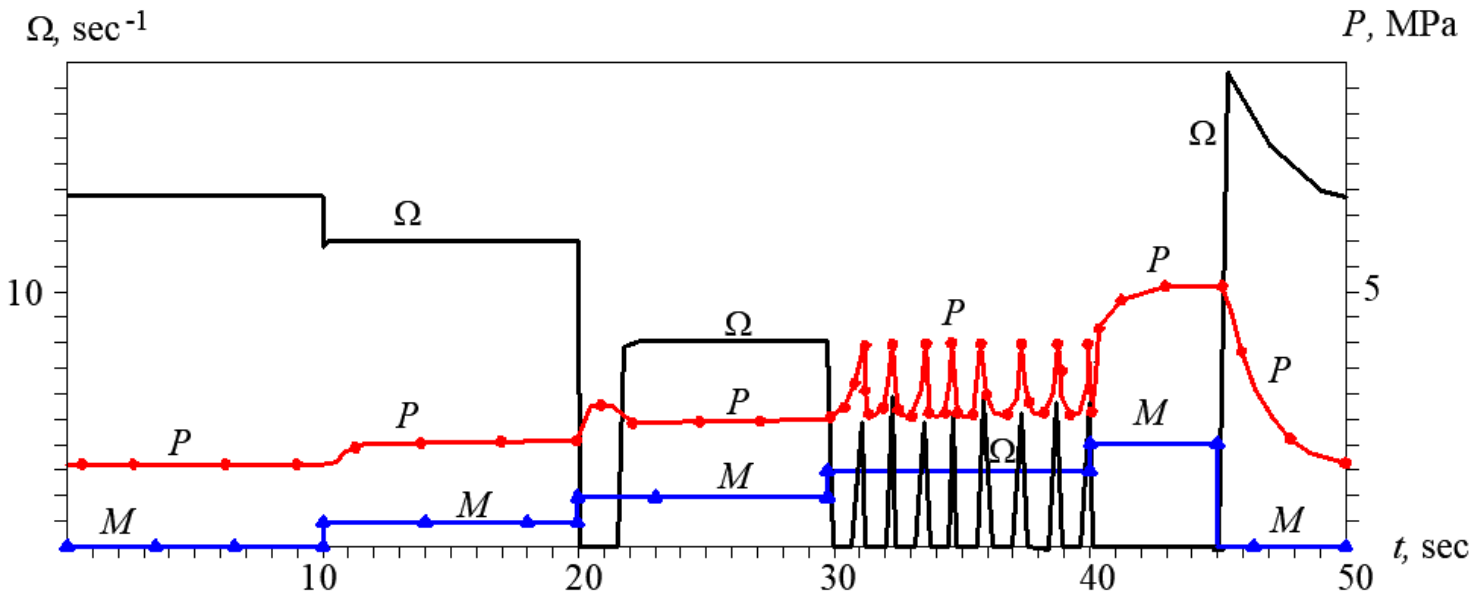

Fig. 3. Calculation oscillogram of the work of the volumetric hydraulic motor with the length of the force line $1500 \mathrm{~m}$

The following is seen from them. At the initial stable operation in the case of a sharp increase in the moment of resistance, the speed $\Omega$ practically instantaneously decreases, and then increases in a fraction of a second to an established value under exponential the law.

One of the forms of stability loss of a given volumetric hydraulic drive, as can be seen from the simulation results, are self-oscillations with a stop. Such a regime is generally characteristic of volumetric hydraulic machines operating at low revs. The experience of the operation of the mentioned devices testifies to their frequent operation in the auto-oscillation mode.

Numerical simulation and operational experience of such systems have shown that the above considerations on the possibility of loss of stability of systems with distributed parameters are justified. In connection with this, a nomogram was constructed for hydraulic systems (Fig. 4), from which it is possible to estimate zones of stable and unstable work. 
International Journal of Mathematical, Engineering and Management Sciences

Vol. 3, No. 4, 315-334, 2018

https://dx.doi.org/10.33889/IJMEMS.2018.3.4-023

We would like to note that the loss of stability for the drive in question is not a catastrophe. When working in the auto-oscillation mode, a rapid enough decrease in the operational capability of both the hydraulic motor itself and the equipment adjacent to it occurs. These features lead to a reduction in a resource of the mechanism. In addition, such a regime affects the quality of the work performed.

In numerical studies in systems with solid-state lines, when they fall into zones where the function $Z(\alpha)<0$, the features of stability loss consisted in stopping the count with a sudden increase in stresses (Kondratenko and Mironova, 2018; Kondratenko et al., 2018). Apparently, in this case there is a loss of forms of oscillation.



Fig. 4. Calculated stability regions (shaded) and instabilities for volumetric hydraulic drives 
International Journal of Mathematical, Engineering and Management Sciences

Vol. 3, No. 4, 315-334, 2018

https://dx.doi.org/10.33889/IJMEMS.2018.3.4-023

\section{Conclusion}

The performed studies have shown that two-link systems with long lines in the process of operation can spontaneously lose stability. The reason is its own dynamic properties, as well as external or internal (for the system) cyclic influence. To exit the zone of instability, the system input should be given a signal that forces a change in the operating mode.

\section{References}

Adamenko, A. I. \& Kondratenko, L. A. (2001). Effect of the depth of the well on the dynamics of screw downhole motors. Journal of Problems of Machine Building and Machine Reliability, 6, 28-33.

Babakov I. M. (1958). Theory of oscillations [in Russian]. GTTL, Moscow

Berezansky, Y. M., \& Kondratiev, Y. G. (2013). Spectral methods in infinite-dimensional analysis (Vol. 12). Springer Science \& Business Media.

Besekerskij, V. A. (1972). Collection of problems on the theory of automatic regulation and control. FML. The Science, Moscow.

Bolotin, V. V. (1956). Dynamic stability of elastic systems [in Russian], Gostekhizdat, Moscow.

Chetaev, N. G. (1950). Stability of motion. Gostekhizdat, Moscow.

Godunov, S. K., \& Ryabenkij V. S. (1977). Difference schemes. FML. The Science, Moscow.

Grigolyuk, Eh.I., \& Selezov N. T. (1973). Nonclassical theories of vibrations of rods, plates and shells. VINITI, Moscow.

Guz' A. N., \& Babich, I. Yu. (1985). Three-dimensional theory of stability of deformed bodies. Naukova dumka, Kiev.

Hurwitz, A. (1895). Ueber die Bedingungen, unter welchen eine Gleichung nur Wurzeln mit negativen reellen Theilen besitzt. Mathematische Annalen, 46(2), 273-284.

Ivanov, V. A., Chemodanov, B. K., \& Medvedev, V. S. (1971). Mathematical foundations of the theory of automatic control. High school, Moscow.

Kamke, E. (1959). Handbook of ordinary differential equations. Leipzig.

Kondratenko, L. A. (2005). Vibrations and speed regulation methods of movement of technological objects. MRSU, Moscow.

Kondratenko, L. A. (2008). Calculation of movement speed variations and stresses in machine assemblies and components. Sputnik, Moscow.

Kondratenko, L. A., \& Mironova, L. I. (2018). Imitation of nonlinear drives with distributed parameters of power lines. Journal of Engineering \& Automation Problems, 1, 92-97.

Kondratenko, L. A., Terechov, V. M., \& Mironova, L. I. (2018). Loss of stability of the drives of rotation with long steel force lines. Journal of Engineering \& Automation Problems, 2, 40-46.

Lur'e A. I. (1970). Theory of elasticity [in Russian]. Nauka, Moscow.

Lyapunov, A. M. (1992). The general problem of the stability of motion (A. T. Fuller Trans.). Taylor \& Francis, London.

Mechanical Engineering (2000). Fedosov E. A. (Chapter Ed.) Encyclopedia [in Russian]. Vol. 1. Moscow. 
International Journal of Mathematical, Engineering and Management Sciences

Vol. 3, No. 4, 315-334, 2018

https://dx.doi.org/10.33889/IJMEMS.2018.3.4-023

Mihaylov A.V. (1939). The theory of stability of linear feedback circuits with lumped parameters. Journal of Technical Physics, 1, 20-31.

Nyquist, H. (1932). Regeneration theory. Bell System Technical Journal, 11(1), 126-147.

Popov, E. P. (1973). Applied theory of control processes in nonlinear systems. Science Pub., Moscow (in Russian).

Postnikov, V. S. (1974). Vnutr. treniye v metallakh (Internal Friction in Metals). Moscow.

Reiner M. Rheology. In Handbuch Der Physik, S. Flugge, Ed., vol. VI, Springer, Berlin, Germany, 1958.

Routh, E. J. (1884). The advanced part of a treatise on the dynamics of a system of rigid bodies. Macmillan and $\mathrm{Co}$, London.

Sedov, L. I. (1970). Continuum Mechanics, Vol. 1. Moscow, SU: Nauka..

Shaginyan, S. G. (2009). On the stability of dynamical systems with distributed parameters for integrally small perturbations. Journal of Works of IPMM NAS of Ukraine, 18, 200-209.

Sirazetdinov, T. K. (1987). Stability of systems with distributed parameters. Nov'osibirsk: Nauka, Sib. Dept, 232. 